\title{
Herbivore Cues from the Fall Armyworm (Spodoptera frugiperda) Larvae Trigger Direct Defenses in Maize
}

\author{
Wen-Po Chuang, ${ }^{1}$ Swayamjit Ray, ${ }^{2}$ Flor Edith Acevedo, ${ }^{3}$ Michelle Peiffer, ${ }^{3}$ Gary W. Felton, ${ }^{3}$ and \\ Dawn S. Luthe ${ }^{2}$ \\ ${ }^{1}$ Department of Entomology, Kansas State University, Manhattan 66506, U.S.A.; ${ }^{2}$ Department of Entomology and \\ ${ }^{3}$ Department of Plant Science, The Pennsylvania State University, University Park 16802, U.S.A.
}

Submitted 10 July 2013. Accepted 3 December 2013.

In addition to feeding damage, herbivores release cues that are recognized by plants to elicit defenses. Caterpillar oral secretions have been shown to trigger herbivore defense responses in several different plant species. In this study, the effects of two fall armyworm (Spodoptera frugiperda) oral secretions (saliva and regurgitant) on caterpillar defense responses in maize (Zea mays) were examined. Only minute amounts of regurgitant were deposited on the maize leaf during larval feeding bouts and its application to leaves failed to induce the expression of several herbivore defense genes. On the other hand, caterpillars consistently deposited saliva on leaves during feeding and the expression of several maize defense genes significantly increased in response to saliva application and larval feeding. However, feeding by ablated caterpillars with impaired salivation did not induce these defenses. Furthermore, bioassays indicated that feeding by unablated caterpillars significantly enhanced defenses when compared with that of ablated caterpillars. Another critical finding was that the maize genotype and stage of development affected the expression of defense genes in response to wounding and regurgitant treatments. These results demonstrate that fall armyworm saliva contains elicitors that trigger herbivore defenses in maize.

To avoid being consumed by insect herbivores, plants have evolved sophisticated mechanisms for recognizing insect cues and triggering defenses against herbivore attack. A cascade of signals are rapidly released upon herbivory that includes ion fluxes, formation of reactive oxygen species, protein modifications, and phytohormone biosynthesis and signaling (Hogenhout and Bos 2011; Mithöfer and Boland 2008; Thivierge et al. 2010). This cascade results in the induction of both direct and indirect defenses against the attacking herbivores (Howe and Jander 2008)

When chewing insects (such as Coleoptera and Lepidoptera species) feed on plants, they cause extensive tissue damage by chewing, snipping, and tearing. However, mechanical damage

Corresponding author: D. S. Luthe; E-mail: ds114@psu.edu; Telephone: +1 (814)863-3542.

* The $\boldsymbol{e}$-Xtra logo stands for "electronic extra" and indicates that two supplementary figures and one supplementary table are published online.

(c) 2014 The American Phytopathological Society alone does not account for the entire range of herbivore effects on plants (Howe and Jander 2008). In addition to damage signals released during feeding, the presence of herbivore cues such as regurgitant and saliva may play a significant role in shaping specific responses to herbivory (Howe and Jander 2008). Large-scale analysis of gene expression has been used to examine the transcriptional differences between mechanical wounding and insect feeding (Major and Constabel 2006; Reymond et al. 2000). These studies revealed that there were differences in the sets of genes expressed in response to mechanical wounding and herbivore attack in Arabidopsis (Reymond et al. 2000). Mechanically wounded tobacco plants also responded differently to the application of water or Manduca sexta regurgitant to the wound site (Halitschke et al. 2003). More than half of the genes that responded to regurgitant had transcriptional changes similar to those of the genes regulated by $M$. sexta feeding, and these changes were attributed to fatty acid-amino acid conjugates present in the regurgitant (Halitschke et al. 2003). The application of regurgitant to wounded tobacco had a greater effect on the production of jasmonic acid (JA) and ethylene (ET), two phytohormones associated with antiherbivore defenses, than wounding alone (Bruinsma and Dicke 2008). Another study reported that the expression of two defense genes (ERF/AP2TF and protease inhibitor) in Arabidopsis was suppressed by regurgitant from caterpillars (Consales et al. 2012). Taken together, these findings indicate that plants elaborate different defense responses to mechanical wounding and herbivore feeding. One of the factors causing these differential responses appears to be insect oral secretions.

Whereas regurgitant arises from the digestive system, saliva is secreted from the salivary glands. In species of Lepidoptera, larvae have two pairs of salivary glands: labial glands and mandibular glands (Bordas 1903; Felton 2008; Parthasarathy and Gopinathan 2005). The long and tubular labial glands secrete watery fluids through the principal secretory structure called a spinneret (Carter and Hargreaves 1986; Felton and Eichenseer 1999; Liu et al. 2004). Mandibular glands are also tubular but their secretions are released from the cutting edge of the mandibles and contain more lipophilic substances such as sterols and triglycerides than the labial glands (Eichenseer et al. 2002; Felton 2008; Howard and Baker 2004; Mossadegh 1978; Wroniszewska 1966). Saliva also may be recognized by plants and further alter plant defenses. This finding was demonstrated by cauterizing (or ablating) the caterpillar spinneret that releases labial saliva (Musser et al. 2002). When ablated Helicoverpa zea caterpillars fed on tobacco, there was more nicotine production 
in plants (Diezel et al. 2009; Musser et al. 2002). This suggested that components of saliva suppress the induction of this defensive secondary metabolite. Similar suppression results were obtained when $M$. sexta regurgitant was applied on tobacco (Kahl et al. 2000). However, it has been shown that glucose oxidase (GOX) from saliva of $H$. zea or European corn borer Ostrinia nubilalis induces defenses in tomato (Louis et al. 2013; Tian et al. 2012). GOX is the most abundant protein (33\%) in the labial saliva of H. zea (Tian et al. 2012) and is found in many caterpillar species (Eichenseer et al. 2010). Other caterpillar salivary proteins, such as ATPase or apyrase, are known to suppress defenses, presumably through their action on extracellular ATP release by plants during wounding (Wu et al. 2012).

Although it is well known that insect saliva can alter plant defenses in dicot species, there is little information regarding the effects of caterpillar saliva on the alteration of direct herbivore defenses in monocots. Although it has been shown that saliva from a member of the stalk-boring Crambidae family, the European corn borer, induced a defense response in maize, the work reported here focuses on the fall armyworm (FAW, Spodoptera frugiperda). Because this herbivore is a member of the Noctuidae family and primarily feeds in maize whorl, it is possible that its oral cues have different effects on maize than those of the European corn borer. This study also examined the effects of FAW oral secretions on the expression of a set of herbivore defense genes from two maize genotypes at different stages of plant development.

\section{RESULTS}

Caterpillars infrequently regurgitate on maize leaves.

It has been shown that plants recognize elicitors from insect regurgitant that trigger plant defenses against herbivores (Howe and Schaller 2008). However, there is a disagreement on whether caterpillars actually regurgitate during most feeding bouts (Peiffer and Felton 2009). Thus, to test whether FAW larvae regurgitate on maize, we first fed larvae an artificial diet containing a fluorescent dye. Then, larvae were allowed to feed on the leaves of two maize inbreds ('Tx601' and 'Mp708'). 'Tx601' is susceptible to FAW feeding (Williams et al. 1989), whereas'Mp708' is highly resistant to a number of lepidopteran pests, including FAW (Williams et al. 1990). The
FAW-fed leaves were then examined for the deposition of regurgitant using confocal fluorescence microscopy. Only a few, faintly fluorescent spots were detected on the leaves of both genotypes (Fig. 1). This was in sharp contrast to the strong fluorescence intensity of regurgitant directly collected from larvae (Fig. 1). Thus, in only 5 to 7 instances did larvae deposit regurgitant among the 15 leaves examined (Table 1). A previous study 'B73' indicated that FAW larvae deposited approximately $1.2 \mathrm{nl}$ of regurgitant per spot on the maize inbred 'B73' during feeding (Peiffer and Felton 2009). Taken together, these results indicated that only minute amounts of regurgitant were deposited on approximately one-third to one-half of the leaf samples. This implies that FAW larvae do not routinely deposit copious amounts of regurgitant on maize plants while feeding. This result was similar to the findings for several other caterpillar species (Peiffer and Felton 2009; Louis et al. 2013).

\section{FAW regurgitant and defense-related genes in maize.}

Although very little regurgitant was detected on maize leaves, we determined whether its application to wounded leaves could induce the expression of several herbivore defense genes. Although it has been reported that regurgitant can trigger direct defenses in maize (Ton et al. 2007), the authors used detached shoots of young seedlings that were placed in the regurgitant solution to determine its effects. In this study, regurgitant was collected from FAW larvae that were fed with either artificial diet or maize leaves (Tx601 inbred); then, $20 \mu \mathrm{l}$ of the regurgitant mixture was applied to each of the ten wound sites per plant (Tx601 at the V7 stage) for $24 \mathrm{~h}$. The expression of three defense genes-MPI, OPR2, and RIP2 — was analyzed by quantitative real-time polymerase chain reaction (qRT-PCR). Maize proteinase inhibitor (MPI), inducible by wounding or caterpillar

Table 1. Numbers of fluorescent spots detected on fall armyworm-fed leaves $^{\mathrm{a}}$

\begin{tabular}{lcc}
\hline Leaves & Tx601 & Mp708 \\
\hline Fall armyworm-fed & $5 / 15$ & $7 / 15$ \\
Control & $0 / 2$ & $0 / 2$ \\
\hline
\end{tabular}

a The numerator represents the total number of fluorescence spots detected and the denominator represents the number of leaves subjected to fall armyworm feeding $(n=15)$ or control leaves $(n=2)$ with no insect feeding.

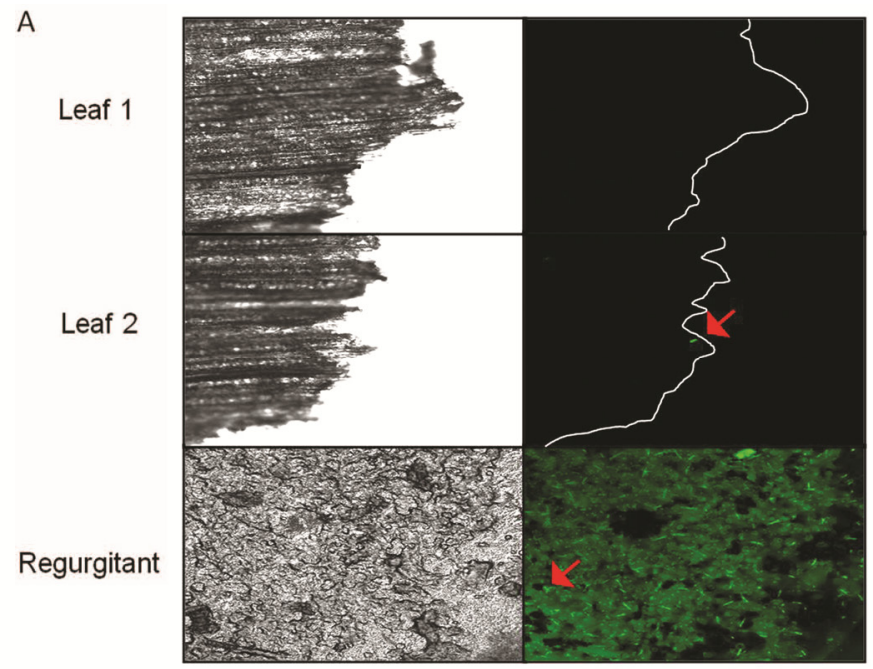

Light microscopy

Fluorescence microscopy
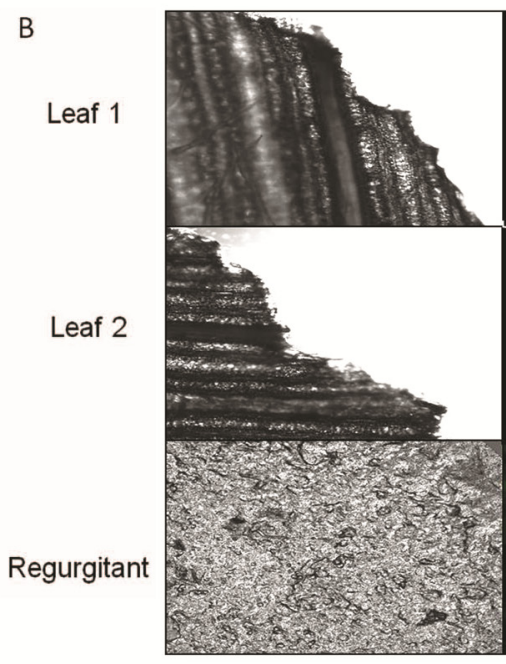

Light microscopy

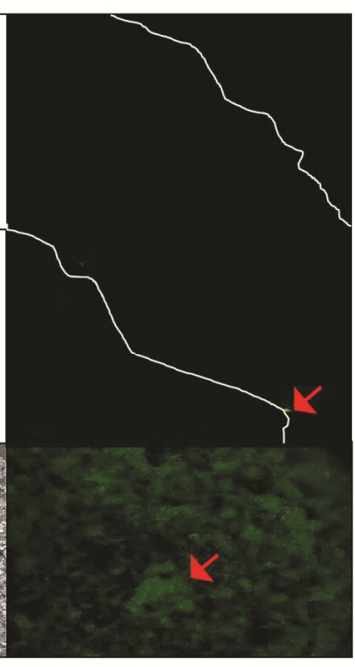

Fluorescence microscopy

Fig. 1. Use of fluorescence microscopy to detect regurgitant on maize leaves during fall armyworm (FAW) feeding on A, Tx601 and B, Mp708 maize leaves. Following larval feeding, leaves were examined under light and fluorescence microscopy. No fluorescence could be detected on leaf 1 (upper panel) and one green fluorescence spot was detected on leaf 2 (middle panel). Fluorescently labeled regurgitant from FAW larvae was the positive control (lower panel). Jagged white lines are traces of the edge of the leaf where feeding occurred and red arrow indicates the fluorescence where regurgitant was deposited. 
feeding, has been shown to inhibit elastase and chymotrypsinlike enzymes in the midgut of $S$. littoralis larvae and play a defensive role against insect herbivores (Tamayo et al. 2000). 12Oxophytodienoic acid-10,11-reductase (OPR2) is involved in the JA-biosynthetic pathway and catalyzes the conversion of 12 oxo-phytodienoic acid to 3-oxo-2-(2-(Z)-pentenyl)-cyclopentane-1 octanoic acid (OPC-8:0). OPC is further processed to become JA. Ribosome-inactivating protein 2 (RIP2) is involved in maize direct defenses (Bass et al. 1992; Chuang et al. 2014). The data showed that the expression of OPR2 and RIP2 of regurgitant-treated plants was not different than that of plants that were wounded and treated with buffer (Fig. 2). Furthermore, the results showed that the source of caterpillar regurgitant (diet or plant fed) did not affect the defense responses. Regurgitant from FAW larvae previously fed on maize leaves did significantly increase the expression of MPI but its application failed to induce the other two defense genes tested.

To determine whether the maize genotype or developmental stage affected the response to regurgitant application, regurgitant was applied to 'B73' and 'Tx601' inbreds at both the V1 and V7 stages (Supplementary Figs. S1 and S2). The V1 seedlings were used because this is the stage typically tested in many maize-herbivore studies such as those reported by Ton and associates (2007). The V7 or midwhorl stage is when plants typically encounter FAW in the southern United States and is the stage that was used for resistance selection in the field (Williams et al. 1989). In this experiment, we tested the expression of the direct defense gene MPI and two terpene synthase genes implicated in indirect defense, TPS10 and TPS23 (Köllner et al. 2008). The data showed that regurgitant applied to V1-stage 'B73' leaves induced TPS10 and MPI but not TPS23 gene expression at $4 \mathrm{~h}$; however, neither TPS 10 nor TPS23 transcripts could be detected at $24 \mathrm{~h}$. In 'B73' at $24 \mathrm{~h}$, the level of MPI transcript induction following treatment with regurgitant was not significantly different than the control. The pattern of regurgitant-induced gene expression in 'Tx601' differed from that of 'B73'. There was no significant difference between control- and regurgitant-treated plants for TPS10 expression at $4 \mathrm{~h}$ in 'Tx601' at the V1 stage. TPS23 transcripts were significantly induced by regurgitant in V1 stage 'Tx601' at $4 \mathrm{~h}$. Neither TPS10 nor TPS23 transcripts were detected in V1 stage 'Tx601' at 24 h. In V1 stage 'Tx601', MPI transcript levels increased in response to regurgitant but the levels of induction were not significantly different between the phosphate-buffered saline (PBS) control and regurgitant-treated samples at both 4 and $24 \mathrm{~h}$. Thus, when 'B73' plants are in the small seedling stage, it appears that regurgitant application can induce the expression of TPS10 and MPI after 4 but not $24 \mathrm{~h}$. In 'Tx601', the only regurgitant-induced gene expression was that of TSP23 at $4 \mathrm{~h}$.
Additional experiments were conducted when 'B73' and 'Tx601' plants were at the V7 stage. The only gene that was significantly upregulated by regurgitant application in this case was TPS10 in 'B73' plants at $4 \mathrm{~h}$. Taken together, these results indicate that the induction of defense gene expression in response to regurgitant is dependent upon plant age, genotype, and time of exposure to regurgitant.

\section{Caterpillars salivate on maize leaves.}

Because it has been demonstrated that many caterpillars, including FAW larvae, contain GOX in their saliva (Eichenseer et al. 2010), we used this protein as marker to determine whether larvae routinely deposit saliva on leaves during feeding. Following FAW feeding, leaf surface was blotted onto a nitrocellulose membrane using a tissue-printing method (Peiffer and Felton 2005) and GOX distribution was determined by immunoblot analysis using anti-GOX antibody. When this technique was used with tomato, it was possible to detect a minimum of $1 \mu \mathrm{g}$ of GOX per leaf (Peiffer and Felton 2005). As a control, we cauterized or ablated the FAW spinneret to block the secretion of saliva to determine whether GOX comes from labial gland (Musser et al. 2006). In 'Tx601' (Fig. 3A), microgram levels of GOX were detected around the caterpillar feeding sites on maize leaves but no GOX was detected on the control leaves. Very little GOX was detected on leaves that were fed on by larvae with ablated spinnerets. We also examined the insect-resistant maize inbred (Mp708) to determine whether saliva was deposited on its leaves (Fig. 3B). Again, GOX was detected near the caterpillar feeding sites but not on the control leaves (Fig. 3B). In addition, more GOX was detected on the plants fed on by unablated than ablated larvae (Fig. 3B). For both maize genotypes, small amounts of GOX were detected on maize leaves that were fed on by ablated larvae (Fig. 3B). The low levels of GOX could be from the mandibular glands (Eichenseer et al. 1999). Alternatively, ablation of the FAW spinneret may not have been $100 \%$ successful in preventing salivation. Nevertheless, these data indicate that FAW larvae routinely deposit their GOX-containing saliva on maize leaves during feeding and that ablation largely prevents the release of labial saliva.

\section{Caterpillar saliva induces}

plant defense-related genes in maize.

Because initial attempts to collect saliva from the FAW spinneret were unsuccessful, scanning electron microscopy (SEM) was used to examine its structure (Fig. 4). Unlike the corn earworm that has a tubular spinneret with a spigot-like structure (Fig. 4A) that allows droplets of saliva to be collected (Tian et al. 2012), the FAW spinneret is a scroll-like structure with a connection on the tip (Fig. 4B and C). This unusual structure

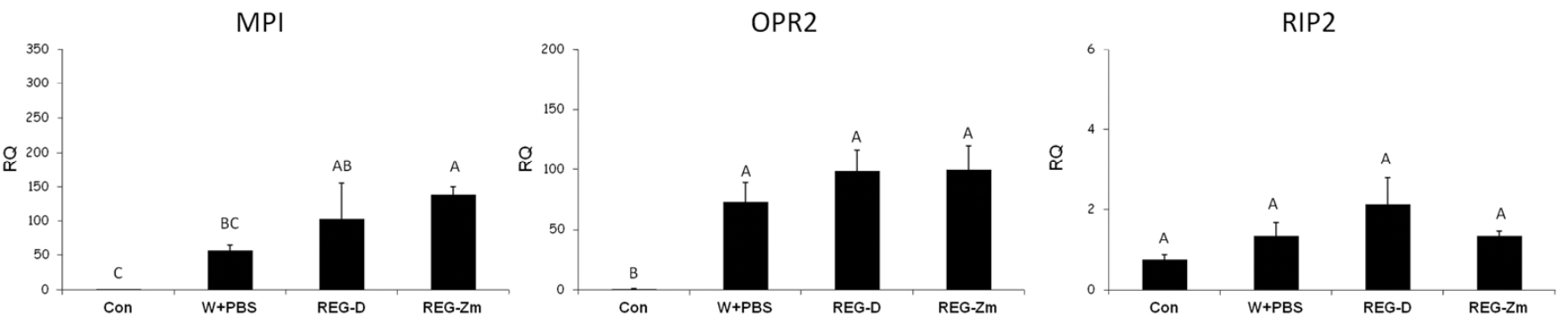

Fig. 2. Expression analysis of maize herbivore defense genes in response to treatment with fall armyworm (FAW) larval regurgitant. Plants at the V7 stage were mechanically damaged using the wounding tool and treated for $24 \mathrm{~h}$ with phosphate-buffered saline (PBS) (W+PBS) or regurgitant from FAW fed on a diet (REG-D) or FAW fed on maize (REG-Zm). Undamaged plants were the control. Total RNA was isolated from tissue near the wounding sites. Relative expression levels (RQ) were determined by quantitative real-time polymerase chain reaction in each biological sample and normalized by to the expression of actin $(n \approx 3$ to 4 , error bar indicates standard error). Letters indicate significant differences as determined by the least significant difference test $(P<0.05)$. $\mathrm{MPI}=$ maize protease inhibitor, OPR2 = 12-oxophytodienoic acid-10,11-reductase and RIP2 = ribosome-inactivating protein 2. 
might explain why it was technically challenging to collect FAW saliva. Despite these difficulties, saliva was collected from the dorsal side (Fig. 4D) and directly applied to wounded leaves to determine whether it affected the expression of the maize defense genes MPI, OPR2, and RIP2. RIP2 is a toxic protein that has been reported to be induced by FAW feeding in maize (Chuang et al. 2014). When saliva was collected from FAW larvae fed on maize and applied to wound sites on V7- stage 'B73' plants, qRT-PCR analysis showed that the transcript abundance for all three genes was significantly higher when the plants were treated with saliva (Fig. 5).

\section{Ablated FAW larvae failed}

to induce plant defense-related genes in maize.

In addition to applying saliva directly to maize leaves, we compared the effects of $24 \mathrm{~h}$ of feeding by ablated and un-

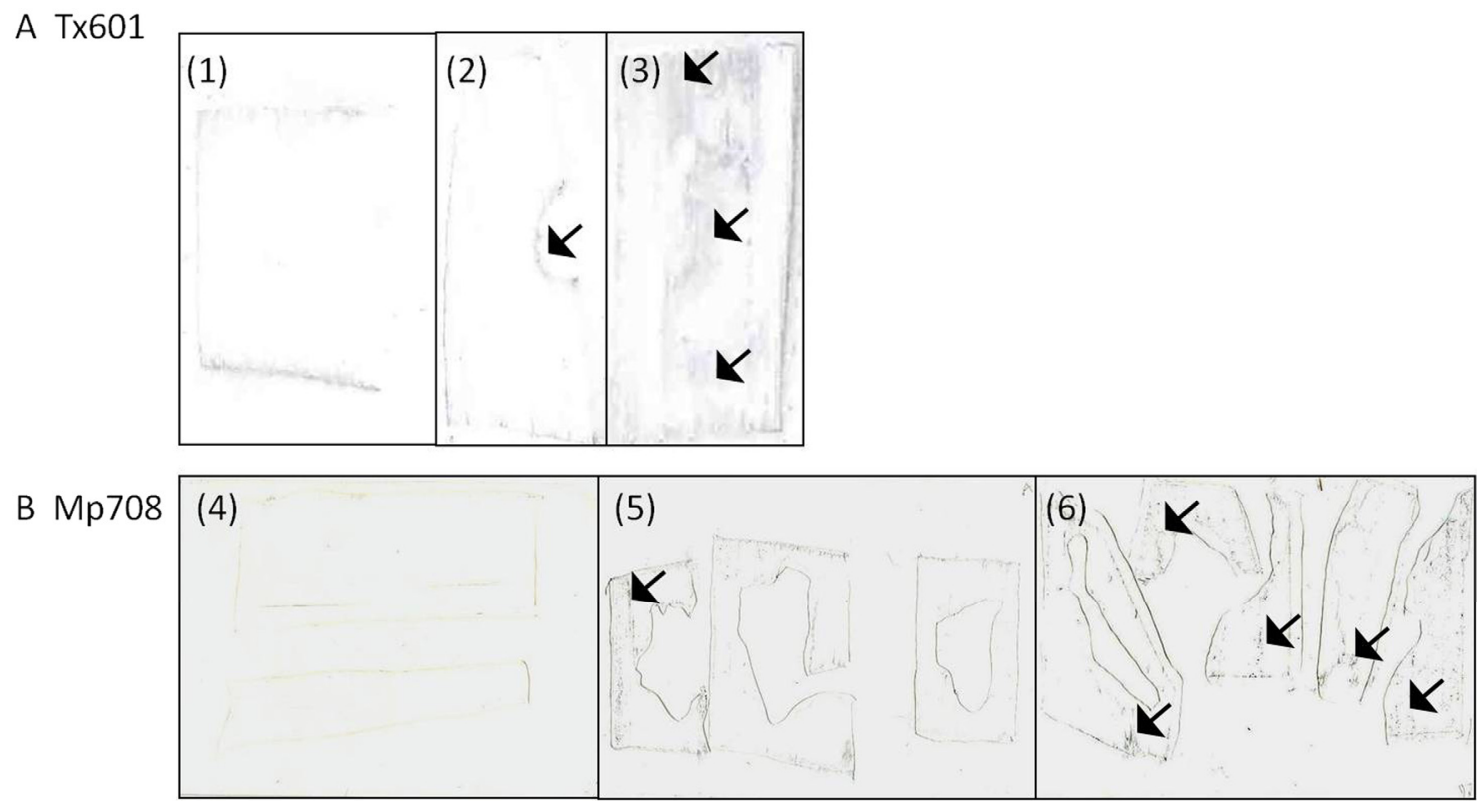

Fig. 3. Immunoblot visualization of glucose oxidase (GOX) deposition on A, Tx601 and B, Mp708 maize leaves. Samples included leaves without fall armyworm (FAW) feeding (panels 1 and 4), leaves with feeding by ablated (panels 2 and 5), and unablated (panels 3 and 6) FAW larvae. Following feeding, leaves were blotted onto a nitrocellulose membrane and leaf shapes were traced with pencil (panels 4 to 6). The membrane was incubated with anti-GOX antibody and immunoreacting spots were visualized colorimetrically. Black dots indicating GOX deposition (marked with arrows) were abundant near the feeding sites.

B

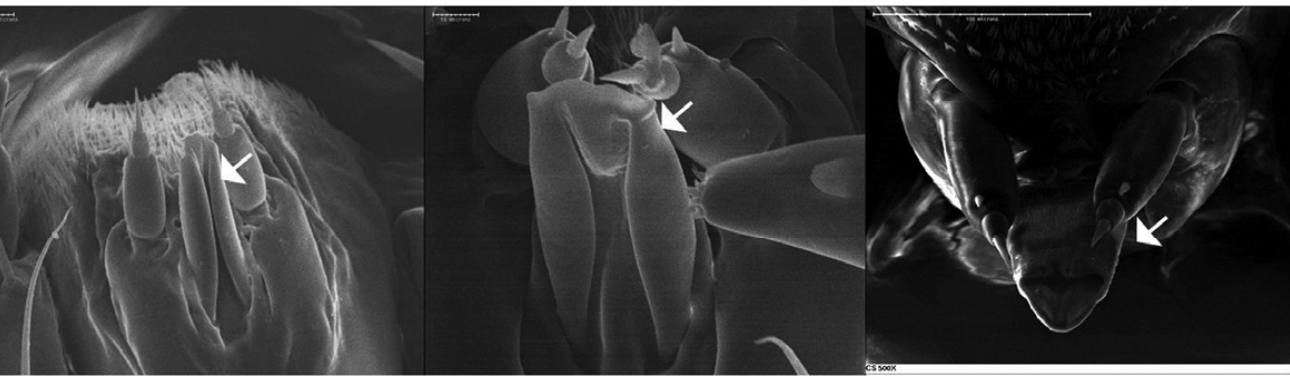

\section{C}

A

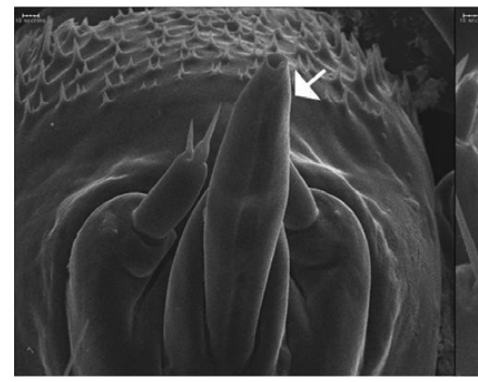

D views of the FAW spinneret. D, Dorsal view of the FAW spinneret. Scale bar $=10 \mu \mathrm{m}$. White arrows indicate the larval spinnerets.
MPI

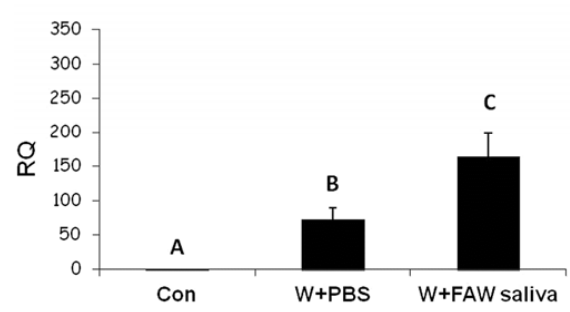

OPR2

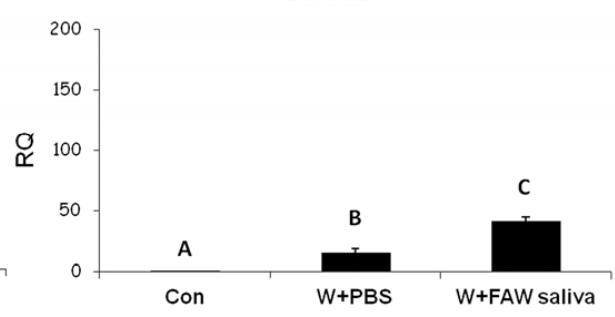

RIP2

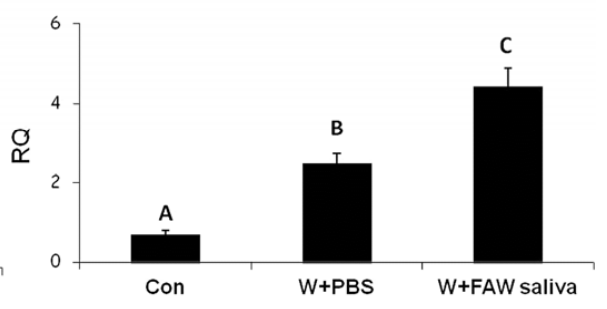

Fig. 5. Expression analysis of maize herbivore defense genes in response to treatment with fall armyworm (FAW) saliva. Plants at the V7 stage were mechanically damaged using the wounding tool and treated for $24 \mathrm{~h}$ with phosphate-buffered saline (PBS) (W+PBS) or with FAW saliva (W+FAW saliva). Undamaged plants were the control. Total RNA was isolated from tissue near the wounding sites. Relative expression levels (RQ) were determined by quantitative real-time polymerase chain reaction in each biological sample and normalized by to the expression of actin $(n=4$, error bar indicates standard error). Letters indicate significant differences as determined by the least significant difference test $(P<0.05)$. MPI $=$ maize protease inhibitor, OPR2 $=12$ oxophytodienoic acid-10,11-reductase, and RIP2 = ribosome-inactivating protein 2. 
ablated FAW larvae on the expression of several genes involved in JA biosynthesis and direct defenses in maize. In this experiment, we examined the expression of the same genes tested in the regurgitant experiment (Fig. 2) and several other genes that function in the JA-biosynthetic pathway or direct defenses. These included phospholipase D that hydrolyzes phospholipids to release linolenic acid from the chloroplast membrane (Shivaji et al. 2010; Wang et al. 2000), allene oxide synthase that catalyzes the first committed step in the JAbiosynthetic pathway (Schaller and Stintzi 2009), and woundinduced protein 1, a Bowman-Birk type proteinase inhibitor that responds to wounding in maize coleoptiles (Rohrmeier and Lehle 1993). As a negative control, expression of a maize aquaporin (PIP1-1) was measured. The results indicated that unablated caterpillars capable of releasing saliva triggered the highest expression of JA-biosynthetic pathway and herbivore defense genes compared with the other treatments (Fig. 6). Although feeding by the ablated caterpillars slightly increased defense gene expression, the induction was not statistically different from control or wounded plants. These results support the data demonstrating that application of FAW saliva elicits herbivore defense gene expression in maize.

\section{Performance of larvae on previously fed maize leaves.}

Although the expression of defense-related genes in maize leaves was induced in response to FAW saliva (Figs. 5 and 6), increased transcript accumulation does not necessarily mean that they are translated into functional proteins that mediate insect resistance. To determine whether effective insect defenses were triggered by caterpillar saliva, plants were infested with ablated or unablated larvae for $24 \mathrm{~h}$, as previously described, and then fed to naïve, neonate FAW larvae to determine whether saliva induced defenses that could impair FAW larval growth. Both the insect-susceptible (Tx601) and -resistant (Mp708) inbreds were tested. In 'Tx601', naïve larvae that fed on plants treated with unablated larvae had the lowest larval weight (Fig. 7A). Naïve larvae that fed on plants treated with Naïve larvae that fed on'Mp708' showed the same trends as those fed on 'Tx601' (Fig. 7B). These data indicated that larvae reared on plants that had their defenses triggered by the feeding of unablated larvae had significantly lower weights compared with those reared on plants that were treated with ablated larvae or control plants. Furthermore, leaves from both genotypes infested with unablated larvae retarded caterpillar growth by approximately $25 \%$ when compared with control plants (Fig. 7A and B). The results also indicated that saliva induced a defense response in both insect-susceptible and insect-resistant inbreds. To ensure that there was no difference in the amount of prior feeding damage between ablated and unablated larvae, we measured the amount of leaf area consumed from both maize genotypes (Fig. 7C and D). The leaf feeding areas were not statistically different between two treatments within the same maize inbred. Thus, this result excludes the possibility that ablated larvae eat less due to damage to the spinneret and attenuate the defense response. These data indicate that maize plants respond to elicitors in caterpillar saliva and mobilize direct defenses against insect herbivory.

\section{DISCUSSION}

Plants can distinguish damage between insect herbivory or mechanical wounding by perceiving herbivore-associated molecular patterns (HAMPs) from insects (Felton and Tumlinson 2008; Mithöfer and Boland 2008). HAMPs are the herbivore equivalent of the microbial-associated molecular patterns (MAMPs) that are well-known molecules or proteins involved in plant-pathogen interactions (Felton and Tumlinson 2008). In contrast to MAMPs, only a few HAMPs have been identified. HAMP proteins include GOX from corn earworm (Eichenseer et al. 1999; Tian et al. 2012), alkaline phosphatase from whitefly (Funk 2001), $\beta$-glucosidase from white cabbage butterfly (Mattiacci et al. 1995), and lipase from grasshopper (Schäfer et al. 2011). In addition, low-MW HAMPs include fatty acid-amino acid conjugates from multiple insect species, inceptins (proteolytic fragments of chloroplastic ATP synthase $\gamma$-subunit) from FAW fed on cowpea or maize (Alborn et al. 1997, 2007; Doss et al. 2000; Schmelz et al. 2006; Yoshinaga et al. 2007), bruchins from pea weevil and cowpea weevil
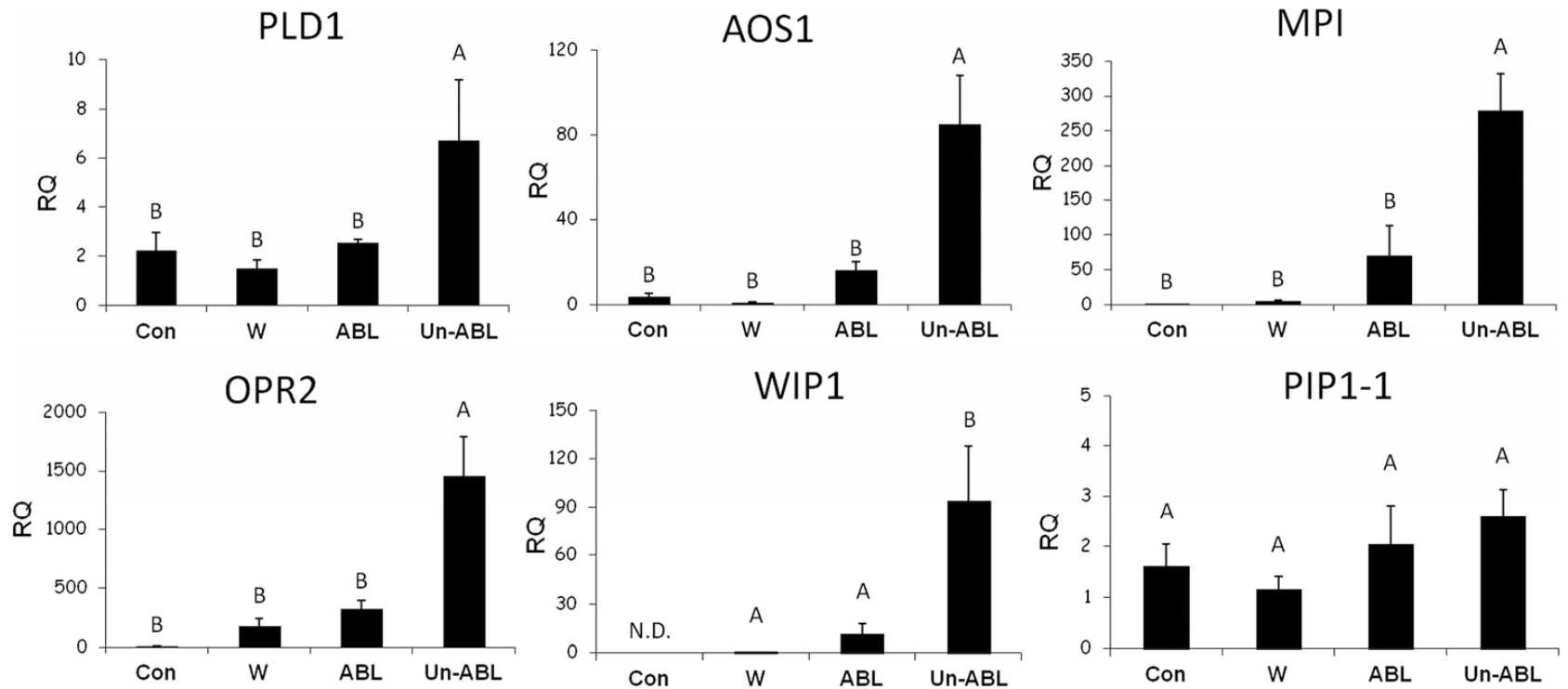

Fig. 6. Expression analysis of maize genes involving in jasmonic acid biosynthesis and herbivore defense in response to wounding (W) and feeding by ablated $(\mathrm{ABL})$ and unablated (Un-ABL) fall armyworm larvae for $24 \mathrm{~h}$. Leaves of plants at the V7 stage were wounded with a paper punch and undamaged plants were the control (Con). Total RNA was isolated from the damaged areas on the leaves. Relative gene expression levels (RQ) were determined by quantitative real-time polymerase chain reaction in each biological sample and normalized to actin gene expression ( $n=4$, error bar indicates standard error). Letters indicate significant differences by least significant difference test $(P<0.05)$. PLD1- phospholipase 1 , AOS $1=$ allene oxide synthase 1 , MPI - maize protease inhibitor, OPR2 = 12-oxophytodienoic acid-10,11-reductase, WIP1 = wound induced protease inhibitor 1 , and PIP1-1 = maize aquaporin. 
(Doss et al. 2000), and caeliferin from grasshopper (Alborn et al. 1997, 2007; Doss et al. 2000; Mithöfer and Boland 2008; Schmelz et al. 2006; Yoshinaga et al. 2007). Although these HAMPs have been identified, there are no HAMPs that induce JA- and ET-related responses in all plant species ( $\mathrm{Wu}$ and Baldwin 2010). Thus, HAMPs seems to be specific to the host plant.

Because some low-MW HAMPs have been identified in the regurgitant of lepidopterans, it has been proposed that, when caterpillars feed on plants, they not only create wounding sites but also release low-MW HAMPs by regurgitation. Thus, plants activate their defense system by recognition of these HAMPs. However, it has been shown that some species of caterpillars do not regurgitate during most feeding bouts (Peiffer and Felton 2009). We used the same fluorescent labeling method to determine whether FAW larvae regurgitated on maize and found that very little regurgitant was deposited on the leaves of two inbreds differing in susceptibility to FAW feeding (Table 1). This result was similar to the previous study using another maize inbred, 'B73' (Peiffer and Felton 2009). These data suggest that FAW larvae do not routinely regurgitate during feeding on maize. Although HAMPs (e.g., inceptins [Schmelz et al. 2006] and volicitin [Spiteller et al. 2001]) have been reported in FAW regurgitant, we did not observe a universal induction of genes involved in direct and indirect defenses when maize plants were treated with regurgitant. As reported by others (Engelberth et al. 2004; Schmelz et al. 2001, 2009), the plants were more responsive to regurgitant application in the V1 seedling stage and the expression of only one gene (TSP10) was induced by regurgitant application to 'B73' in the V7 stage. This could be due to changes in the cuticular wax composition from long-chain alcohols and aldehydes to esters that occurs when maize undergoes the developmental transition from the juvenile to adult stage (Lawson and Poethig 1995).

Another potential source of FAW HAMPs is saliva. Caterpillar saliva has lubrication, predigestion, and antimicrobial functions (Riberiro 1995). However, plants also may recognize HAMPs in saliva and respond by triggering plant defenses (Tian et al. 2012). Immunoblot analysis showed that FAW larvae consistently deposited the secreted salivary enzyme GOX through the spinneret onto wounds on the maize leaf (Fig. 3). Unlike tomato (Tian et al. 2012), there was no defense gene induction when maize plants were treated with fungal GOX (Louis et al. 2013). This has been attributed to the fourfold lower levels of glucose measured in maize leaves compared with tomato (Louis et al. 2013). The lack of sufficient substrate could limit GOX activity in maize. In fact, it has been shown that the application of GOX plus glucose triggers defense gene expression in maize (Louis et al. 2013). This suggests that some other components of saliva are involved in defense induction in maize.

In this study, data from gene expression analyses and bioassays clearly indicated that FAW saliva plays an important role in eliciting herbivore defenses in maize. Genes involved in the JA-biosynthetic pathway were upregulated by FAW saliva (Figs. 5 and 6), indicating that it could enhance defenses by elevating JA production. Furthermore, maize genes encoding
Tx601

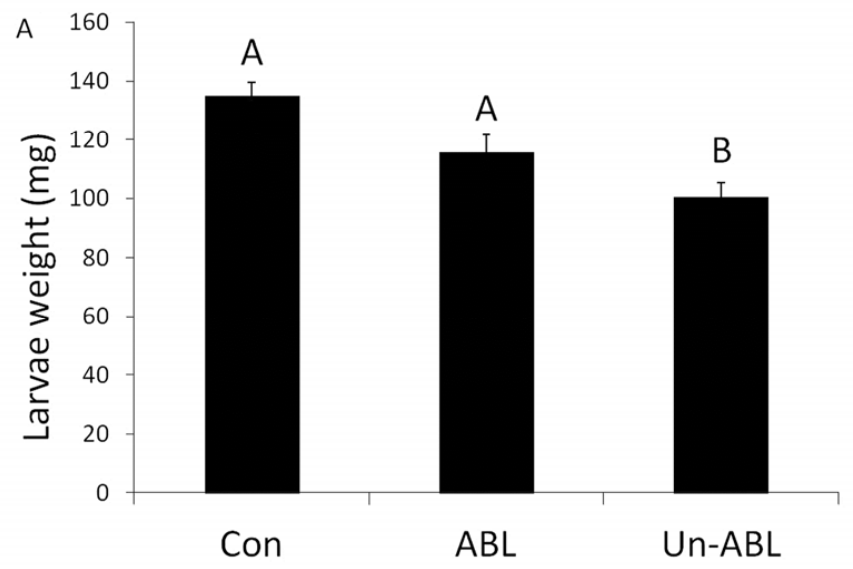

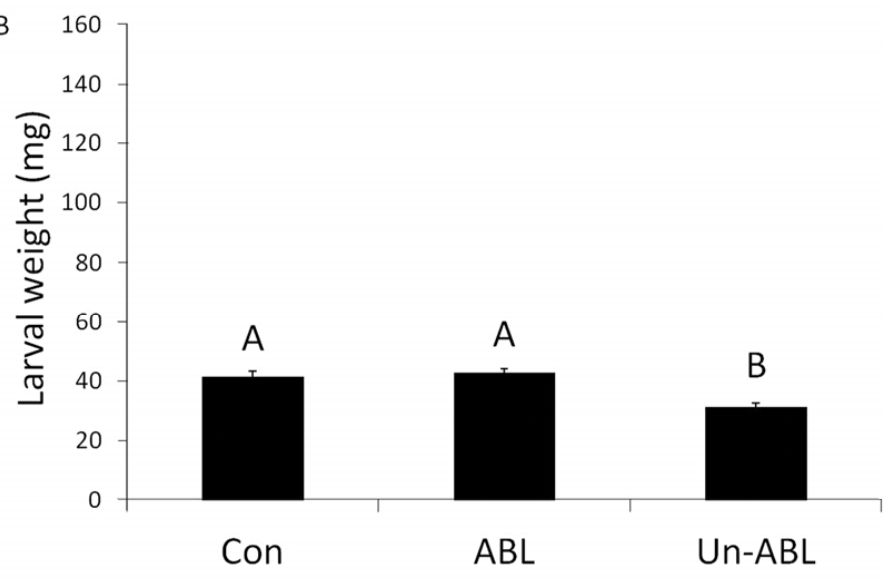

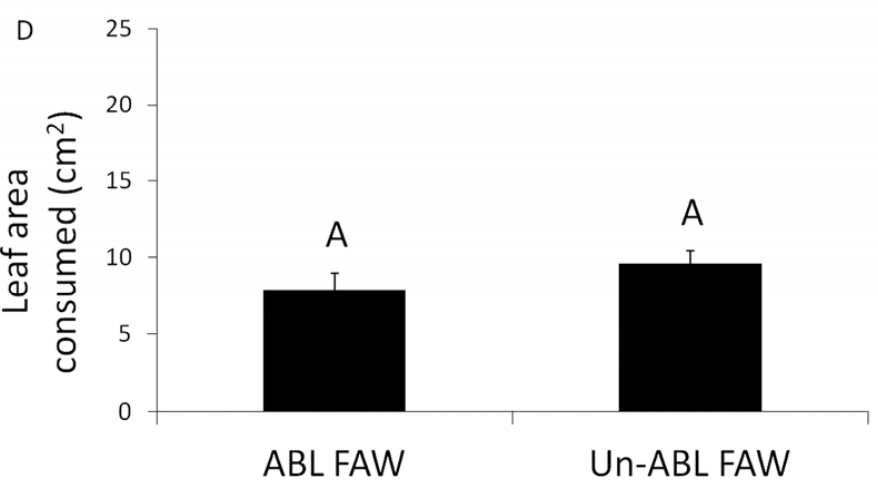

ABLFAW

Mp708

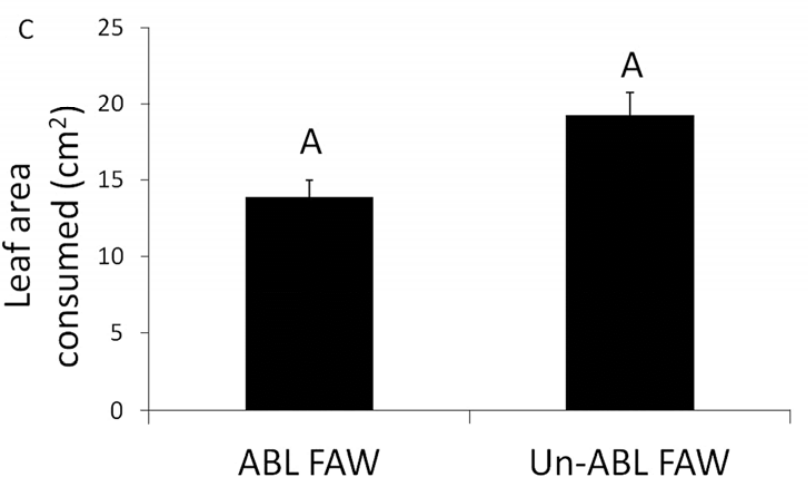

Fig. 7. Performance of naïve fall armyworm (FAW) larvae on maize plants at the V7 stage subjected to feeding by ablated and unablated FAW larvae. A, Tx601 and B, Mp708 plants were infested with ablated and unablated larvae for $24 \mathrm{~h}$; then, leaves were collected for bioassays with naïve larvae (5.0 to $7.5 \mathrm{mg}$ ) for 4 days, when they were weighed. The leaf area $\left(\mathrm{cm}^{2}\right)$ eaten by ablated and unablated larvae was calculated for FAW that fed on $\mathbf{C}$, Tx601 or D, Mp708. Detached leaves were fed to larvae for $24 \mathrm{~h}$ and the leaf area before and after feeding was determined by SigmaScan Pro 5 . Data are represented as area ( $\left.\mathrm{cm}^{2}\right)$ consumed. Letters indicate significant differences determined by the least significant difference test $(P<0.05) ; n=16-28(\mathrm{~A}), n=98-115(\mathrm{~B}), n=10-11$ (C), and $n=9-10(\mathrm{D})$; error bars indicate standard error. 
proteins involved in direct defenses were also induced by FAW saliva (Figs. 5 and 6). Although ablated larvae also induced the JA-biosynthetic pathway and direct defense genes, the induction was similar to mechanically damaged plants. These results demonstrated that ablated larvae that were unaffected in their ability to regurgitate were not able to trigger direct defenses in maize. This was supported by the bioassay data demonstrating that feeding by ablated larvae did not induce sufficient direct defenses to significantly retard larval growth (Fig. 7A and B). Although it could be questioned whether ablated larvae ate less leaf tissue due to spinneret damage, there was no difference between amounts of leaves consumed by ablated larvae and unablated larvae (Fig. 7C and D). In addition, in the geneexpression and bioassay experiments, we placed three larvae on each plant to induce plant defenses and observed that both ablated and unablated larvae seriously damaged the plants. Despite the similar levels of damage, plants responded to the ablated and unablated larvae very differently. These results indicate that saliva from FAW larvae has the capacity to elicit direct defense proteins in maize but the specific eliciting components in the saliva have yet to be identified.

Labial salivary glands in the silk moth Bombyx mori are referred to as silk glands that produce silk proteins in the last stage of larval development. These silk proteins are the most well-known labial saliva proteins (Celorio-Mancera et al. 2011; Mondal et al. 2007). However, the components of labial salivary glands in other species of Lepidoptera have not been completely identified. Recently, the sialome (salivary transcriptome and proteome) and structure of labial salivary glands of $H$. armigera have been reported (Celorio-Mancera et al. 2011; Sorensen et al. 2006). Several digestive proteins (amylase and maltase), lysozymes, proteases, and proteinase inhibitors have been identified from the salivary transcriptome (CelorioMancera et al. 2011). This comprehensive study suggests that labial salivary glands in species of Lepidoptera not only function as silk glands but also secrete proteins involved in predigestion, insect immunity, detoxification, and other unknown functions. Another similar finding was reported from saliva obtained from the closely related species $H$. zea (Tian et al. 2012). Our study provides evidence that saliva from labial salivary glands of FAW also is an important mediator of defenses in maize.

It was technically challenging to collect saliva directly from the spinneret. The spinneret structure of larval B. mori that is used to produce silk fibroin fibers has been extensively studied (Asakura et al. 2006). However, there have been few reports of spinneret structures for other Lepidoptera species (Sorensen et al. 2006). The spinnerets of $H$. zea and H. armigera are tubular, spigot-like structures (Felton 2008; Sorensen et al. 2006). The spinneret base is mushroom-like and the tip region forms a circular structure in H. armigera larvae (Sorensen et al. 2006). Compared with these two insect species, the spinneret structure of FAW larvae is quite different (Fig. 4). The tip of the spinneret is a fixed connection structure between two tubular or scroll-like structures. The saliva could be released from the inner region of two tubular structures or the inner region of the connection structure on the top. This might explain why saliva collection from FAW larvae is difficult. Despite the difficulty, we believed that it was the preferred method for working with saliva because salivary gland homogenates contain hundreds of proteins that are not secreted and, thus, may confound the interpretation of the results.

In summary, we demonstrate that the herbivore cues or HAMPs arise from both saliva and regurgitant of the FAW. However, due to the minute amounts of regurgitant released during feeding and the lack of response of some plant defenses, our data indicate that the major source of HAMPs or elicitors of maize direct defense genes arise from saliva and not from regurgitant. To date, the majority of HAMPs have been identified from the regurgitant of caterpillars; however, in two recent examples, including the FAW and the European corn borer (Louis et al. 2013), saliva appears to be the major source of these HAMPs. Ongoing research in our laboratory is aimed at identifying the HAMPs in FAW saliva.

\section{MATERIALS AND METHODS}

\section{Plant materials and insect rearing.}

Maize (Zea mays) inbreds that are resistant (Mp708) and susceptible ('Tx601' and 'B73') to FAW feeding were obtained from W. P. Williams (United States Department of AgricultureAgricultural Research Service [USDA-ARS], Mississippi State University (Mississippi State, MS, U.S.A.)). Seed were sown in pots filled with topsoil (Hagerstown loam) in the Crop and Soil Sciences greenhouse at The Pennsylvania State University, University Park. Well-watered maize plants were grown under greenhouse conditions until they reached specified developmental stages (V1 and V7) (Ritchie 1986). For experiments that involved wounding, plants were either wounded with a specially designed tool (Bosak 2011) or with a paper punch (6 $\mathrm{mm}$ in diameter, 10 times per plant). The type of wounding used is indicated in each figure legend. Following wounding or caterpillar feeding, tissue adjacent to the damaged areas was excised, frozen in liquid nitrogen, and stored at $-80^{\circ} \mathrm{C}$.

FAW eggs were obtained from USDA-ARS Corn Host Plant Resistance Research Laboratory at Mississippi State University. Larvae were reared on an artificial diet (Peiffer and Felton 2005 ) in a $27^{\circ} \mathrm{C}$ incubator with a 16 -h photoperiod and newly molted fifth-instar larvae were used for all experiments, except for bioassays with neonate larvae.

\section{Detection of FAW regurgitant on maize leaves.}

To determine the release of regurgitant during FAW feeding a method by Peiffer and Felton (2009) was used. Briefly, newly molted fifth-instar FAW larvae were fed with a fluorescent dye (Alexa Fluor 488; Invitrogen, Carlsbad, CA, U.S.A.) on an artificial diet. Larvae were then placed on pieces of insect-susceptible ('Tx601') and insect-resistant ('Mp708') maize leaves and allowed to feed. After several minutes of feeding, leaf material was analyzed for a fluorescence signal using an Olympus FluoView FV1000 (Olympus, Center Valley, PA, U.S.A.) confocal microscope. The data were analyzed by the Olympus FluoView FV1000 software. Regurgitant was collected from the treated insects to confirm the presence of fluorescent dye in their oral secretions. The dye is not taken up by the salivary glands and, thus, is not released in saliva.

\section{FAW regurgitant and its effect on plant defenses.}

FAW regurgitant was collected from FAW fifth-instar larvae fed with artificial diet or maize leaves by gently squeezing the lava until regurgitant was expelled (Tian et al. 2012). An equal amount of regurgitant was mixed with $1 \times$ PBS buffer (regurgitant mixture) and $20 \mu \mathrm{l}$ of regurgitant mixture was applied to each of 10 wound sites per plant. Leaf tissue around the wounding sites was collected at 4 and $24 \mathrm{~h}$ after regurgitant application and defense gene expression was examined by qRT-PCR, as described below.

\section{Tissue printing and immunoblotting} to detect saliva deposition on leaves.

Maize plants were infested with ablated and unablated caterpillar for a few hours until several feeding sites were evident. Fed leaf tissues were immediately blotted onto nitrocellulose 
membranes using the Panther Semi-Dry Electroblotter (Thermo Scientific, Owl, MA, U.S.A.). Undamaged plants were used as controls. Because it is known that FAW saliva contains GOX (Eichenseer et al. 2010), its deposition was detected by immunoblot analysis using anti-GOX antibody, as previously described (Peiffer and Felton 2005).

\section{Ablation of the spinneret to prevent salivation.}

The late fourth-instar FAW larvae were placed on ice until flaccid. The larvae were immobilized with a hair clip and the spinnerets cauterized with a heat pen (Electron Microscopy Sciences, Hatfield, PA, U.S.A.), as described by Peiffer and Felton (2005). The cauterized larvae were placed on an artificial diet overnight to recover and molt. The newly molted fifthinstar ablated and unablated larvae were used to determine the effect of saliva on plant defenses.

FAW saliva and its effect on plant defense gene expression.

Maize plants were infested with ablated and unablated larvae for $24 \mathrm{~h}$. Three larvae were placed in each plant. A paper punch was used to mimic mechanical wounding (multiple wounding sites per plant) and undamaged plants were used as positive and negative controls. Each treatment had four biological replicates. Plant defense gene expression was examined using qRT-PCR.

\section{Application of FAW saliva to maize leaves.}

Because saliva initially proved to be very difficult to collect from FAW larvae compared with $H$. zea larvae, we examined the structure of the spinneret by SEM to aid in our ability to collect saliva. Larvae were washed before fixation by sonication in $1 \%$ Triton X-100 for $30 \mathrm{~s}$. After three rinses in distilled $\mathrm{H}_{2} \mathrm{O}$, larvae were patted dry and prepared as described by Hayat (1989). Briefly, the larval heads were removed and fixed overnight in $2.5 \%$ glutaraldehyde and $1.5 \%$ formaldehyde (Electron Microscopy Sciences) in $0.1 \mathrm{M}$ sodium cacodylate, $\mathrm{pH}$ 7.4. Larvae were then rinsed in $0.1 \mathrm{M}$ sodium cacodylate and treated with $2 \%$ aqueous osmium tetroxide (Electron Microscopy Sciences) for $2 \mathrm{~h}$. Following water rinses, the larvae were dehydrated in graded ethanol solutions (70 to $100 \%$ ), then dried in a Baltec SCD030 (Techno Trade, Manchester, NH, U.S.A.) critical point dryer. Dried samples were mounted and coated with gold or palladium to increase conductivity (Baltec SCD050; Techno Trade). Samples were imaged at $20 \mathrm{KV}$ in a JSM 5400 scanning electron microscope (JEOL, Peabody, MA, U.S.A.), and images were captured using IMIX-PC software (v.10; Princeton Gamma Tech, Princton NJ, U.S.A.).

Following a detailed examination of the spinneret, we were able to collect saliva from FAW. FAW saliva was then collected from 50 sixth-instar larvae reared on detached leaves of the inbred 'B73', as described for $H$. zea larvae (Tian et al. 2012). The saliva was stored in glycerol and kept at $-80^{\circ} \mathrm{C}$. The saliva samples were combined into a single tube $(10 \mu \mathrm{l})$ and $1 \times$ PBS $(70 \mu \mathrm{l})$ was added to obtain a final volume of 80 $\mu \mathrm{l}$ (saliva mixture). The third leaf (from top to bottom) in V7 stage of 'B73' plants was wounded using a wounding tool (Bosak 2011). The saliva mixture $(15 \mu \mathrm{l})$ was applied to each wounding site and the total amount of protein applied to each plant was approximately $3.9 \mu \mathrm{g}$. Leaf tissues around the wounding sites were collected at $24 \mathrm{~h}$ after saliva application to examine defense gene expression by qRT-PCR.

\section{Effect of saliva on plant resistance to FAW larvae.}

To determine whether the deposition of saliva on maize affected plant defense, plants were infested with ablated and unablated FAW larvae for $24 \mathrm{~h}$. Three larvae were placed in each plant and 18 to 30 total plants were infested. The fed leaf tissues were collected, cut into approximately $1-\mathrm{cm}$ pieces, and placed in diet cups. A paper punch was used to mimic mechanical wounding, as described above. Naïve neonate larvae ( 5 to $7.5 \mathrm{mg}$ ) were placed into diet cups with the treated leaf tissues. Each treatment had 30 larvae. Bioassays using the inbred 'Tx601' were done one time and those with'Mp708' were repeated three times. The bioassays were conducted at $26^{\circ} \mathrm{C}$ for 4 days in a 16-h photoperiod before the final larval weights were determined. The results were analyzed by SAS statistical software (SAS, Cary, NC, U.S.A.).

\section{qRT-PCR of defense gene expression.}

Total RNA from leaf tissue was isolated using the TRIzol Reagent (Invitrogen) and DNase (Promega Corp., Madison, WI, U.S.A.) following the manufacturer's instructions. The firststrand cDNA was synthesized with a high capacity cDNA reverse transcription kit (ABI, Foster City, CA, U.S.A.) with oligo (dT) 20 primers following the manufacturer's instructions. qRT-PCR was carried out in an ABI 7500 Fast real time PCR system. The primers were designed by Primer Express software for real-time PCR (version 3.0; ABI). The PCR was conducted by using the default conditions: step $1,50^{\circ} \mathrm{C}$ for $2 \mathrm{~min}$ and $95^{\circ} \mathrm{C}$ for $10 \mathrm{~min}$; step $2,95^{\circ} \mathrm{C}$ for $15 \mathrm{~s}$ and $60^{\circ} \mathrm{C}$ for $1 \mathrm{~min}$ repeated for 40 cycles; step $3,72^{\circ} \mathrm{C}$ for $10 \mathrm{~min}$; and step 4 , dissociation stage. Each qRT-PCR reaction was $10 \mu \mathrm{l}$ and there were three technical replicates for each sample. The relative quantification of gene expression was determined by the ABI 7500 Fast SDS software (version 1.4; ABI). The data set was normalized using an actin gene as a control. Gene-specific forward and reverse primers in this study are listed in Supplementary Table S1. The results were analyzed by SAS statistical software (SAS).

\section{Quantification of FAW feeding damage.}

To determine whether ablation affected the amount of feeding damage, maize leaves $(4$ by $5 \mathrm{~cm}$ ) were detached from the plant, scanned, and placed in a $1 \%$ agar petri dish (VWR International, Radnor, PA, U.S.A.). Fifth-instar larvae (ablated and unablated) were allowed to feed for $24 \mathrm{~h}$ (one larva per leaf); then, leaves were scanned. Scanned files of leaf tissues before and after caterpillar feeding were analyzed by SigmaScan Pro 5 (SPSS Science, Chicago). The feeding area results were analyzed by SAS statistical software (SAS).

\section{ACKNOWLEDGMENTS}

Support by the USDA AFRI program (2010-65105-20639 and 201167013-30352) awarded to G. W. Felton and D. S. Luthe and the National Science Foundation (IOS-0641219 to awarded to D. S. Luthe) made this research possible. We thank Penn State Microscopy and Cytometry Facility (University Park, PA, U.S.A.) for their technical support, P. W. Williams for providing maize seed and, E. J. Bosak for inventing the wounding tool.

\section{LITERATURE CITED}

Alborn, H. T., Turlings, T. C., Jones, T. H., Stenhagen, G., Loughrin, J. H., and Tumlinson, J. H. 1997. An elicitor of plant volatiles from beet armyworm oral secretion. Science 276:945-949.

Alborn, H. T., Hansen, T. V., Jones, T. H., Bennett, D. C., Tumlinson, J. H., Schmelz, E. A., and Teal, P. E. A. 2007. Disulfooxy fatty acids from the American bird grasshopper Schistocerca americana, elicitors of plant volatiles. Proc. Natl. Acad. Sci. U.S.A. 104:12976-12981.

Asakura, T., Umemura, K., Nakazawa, Y., Hirose, H., Higham, J., and Knight, D. 2006. Some observations on the structure and function of the spinning apparatus in the silkworm Bombyx mori. Biomacromolecules 8:175-181.

Bass, H. W., Webster, C., O'Brian, G. R., Roberts, J. K., and Boston, R. S. 1992. A maize ribosome-inactivating protein is controlled by the transcriptional activator Opaque-2. Plant Cell 4:225-234. 
Bordas, L. 1903. Mandibular glands of Lepidoptera larvae. CR Hebd. Acad. Sci. 136:1273-1275.

Bosak, E. J. 2011. Using a developmental comparison to decipher priming of induced defenses in maize and its effects on a generalist herbivore. $\mathrm{PhD}$ dissertation. Department of Entomology, The Pennsylvania State University, University Park, PA, U.S.A. Published online.

Bruinsma, M., and Dicke, M. 2008. Herbivore-induced indirect defense: From induction mechanisms to community ecology. Pages 31-60 in: Induced Plant Resistance to Herbivory. A. Schaller, ed. Springer, Dordrecht, The Netherlands.

Carter, D. J., and Hargreaves, B. 1986. The anatomy of caterpillars. Page 296 in: A Field Guide to Caterpillars of Butterflies and Moths in Britain and Europe. D. J. Carter, ed. Collins, London.

Celorio-Mancera, M. d. 1. P., Courtiade, J., Muck, A., Heckel, D. G., Musser, R. .O., and Vogel, H. 2011. Sialome of a generalist lepidopteran herbivore: Identification of transcripts and proteins from Helicoverpa armigera labial salivary glands. PLoS One 6:e26676. Published online.

Chuang, W.-P. Herde, M., Ray, S., Castano-Duque, L. Howe, G. A., and Luthe, D. S. 2014. Caterpillar attack triggers accumulation of the toxic maize protein, RIP2. New Phytol. 201:928-939.

Consales, F., Schweizer, F., Erb, M., Gouhier-Darimont, C., Bodenhausen, N., Bruessow, F., Sobhy, I., and Reymond, P. 2012. Insect oral secretions suppress wound-induced responses in Arabidopsis. J. Exp. Bot. 63:727-737.

Diezel, C., von Dahl, C. C., Gaquerel, E., and Baldwin, I. T. 2009. Different Lepidopteran elicitors account for cross-talk in herbivory-induced phytohormone signaling. Plant Physiol. 150:1576-1586.

Doss, R. P., Oliver, J. E., Proebsting, W. M., Potter, S. W., Kuy, S., Clement, S. L., Williamson, R. T., Carney, J. R., and DeVilbiss, E. D. 2000. Bruchins: Insect-derived plant regulators that stimulate neoplasm formation. Proc. Natl. Acad. Sci. U.S.A. 97:6218-6223.

Eichenseer, H., Mathews, M. C., Bi, J. L., Murphy, J. B., and Felton, G. W. 1999. Salivary glucose oxidase: Multifunctional roles for Helicoverpa zea? Arch. Insect Biochem. Physiol. 42:99-109.

Eichenseer, H., Murphy, J. B., and Felton, G. W. 2002. Sequestration of host plant carotenoids in the larval tissues of Helicoverpa zea. J. Insect Physiol. 48:311-318.

Eichenseer, H., Mathews, M., Powell, J., and Felton, G. 2010. Survey of a salivary effector in caterpillars: Glucose oxidase variation and correlation with host range. J. Chem. Ecol. 36:885-897.

Engelberth, J., Alborn, H. T., Schmelz, E. A., and Tumlinson, J. H. 2004 Airborne signals prime plants against insect herbivore attack. Proc. Natl. Acad. Sci. U.S.A. 101:1781-1785.

Felton, G. W. 2008. Caterpillar secretions and induced plant responses. Pages 369-387 in: Induced Plant Resistance to Herbivory. A. Schaller, ed. Springer, Dordrecht, The Netherlands.

Felton, G. W., and Eichenseer, H. 1999. Herbivore saliva and its effects on plant defense against herbivores and pathogens. Pages ix and 390 in: Induced Plant Defenses Against Pathogens and Herbivores: Biochemistry, Ecology, and Agriculture. A. A. Agrawal, S. Tuzun, and E. Bent, eds. American Phytopathological Society Press, St. Paul, MN, U.S.A.

Felton, G. W., and Tumlinson, J. H. 2008. Plant-insect dialogs: Complex interactions at the plant-insect interface. Curr. Opin. Plant Biol. 11:457463

Funk, C. J. 2001. Alkaline phosphatase activity in whitefly salivary glands and saliva. Arch. Insect Biochem. Physiol. 46:165-174.

Halitschke, R., Gase, K., Hui, D., Schmidt, D. D., and Baldwin, I. T. 2003. Molecular interactions between the specialist herbivore Manduca sexta (Lepidoptera, Sphingidae) and its natural host Nicotiana attenuata. VI. Microarray analysis reveals that most herbivore-specific transcriptional changes are mediated by fatty acid-amino acid conjugates. Plant Physiol. 131:1894-1902.

Hayat, M. A. 1989. Principles and Techniques of Electron Microscopy: Biological Applications. CRC Press, Boca Raton, FL, U.S.A.

Hogenhout, S. A., and Bos, J. I. B. 2011. Effector proteins that modulate plant-insect interactions. Curr. Opin. Plant Biol. 14:422-428.

Howard, R. W., and Baker, J. E. 2004. Stage-specific surface chemicals of Plodia interpunctella: 2-Acyl-1,3-cyclohexanediones from larval mandibular glands serve as cuticular lipids. Comp. Biochem. Physiol. B Biochem. Mol. Biol. 138:193-206.

Howe, G. A., and Jander, G. 2008. Plant immunity to insect herbivores. Annu. Rev. Plant Biol. 59:41-66.

Howe, G. A., and Schaller, A. 2008. Direct defenses in plants and their induction by wounding and insect herbivores. Pages 7-29 in: Induced Plant Resistance to Herbivory. A. Schaller, ed. Springer, Dordrecht, The Netherlands.

Kahl, J., Siemens, D. H., Aerts, R. J., Gabler, R., Kuhnemann, F., Preston, C. A., and Baldwin, I. T. 2000. Herbivore-induced ethylene suppresses a direct defense but not a putative indirect defense against an adapted herbivore. Planta 210:336-342.

Köllner, T. G., Held, M., Lenk, C., Hiltpold, I., Turlings, T. C., Gershenzon, J., and Degenhardt, J. 2008. A maize (E)- $\beta$-caryophyllene synthase implicated in indirect defense responses against herbivores is not expressed in most American maize varieties. Plant Cell 20:482-494.

Lawson, E. J., and Poethig, R. S. 1995. Shoot development in plants: Time for a change. Trends Genet. 11:263-268.

Liu, F., Cui, L., Cox-Foster, D., and Felton, G. W. 2004. Characterization of a salivary lysozyme in larval Helicoverpa zea. J. Chem. Ecol. 30:2439-2457.

Louis, J., Peiffer, M., Ray, S., Luthe, D. S., and Felton, G. W. 2013. Hostspecific salivary elicitor(s) of European corn borer induce defenses in tomato and maize. New Phytol. 199:66-73.

Major, I. T., and Constabel, C. P. 2006. Molecular analysis of poplar defense against herbivory: Comparison of wound- and insect elicitorinduced gene expression. New Phytol. 172:617-635.

Mattiacci, L., Dicke, M., and Posthumus, M. A. 1995. Beta-Glucosidase: An elicitor of herbivore-induced plant odor that attracts host-searching parasitic wasps. Proc. Natl. Acad. Sci. U.S.A. 92:2036-2040.

Mithöfer, A., and Boland, W. 2008. Recognition of herbivory-associated molecular patterns. Plant Physiol. 146:825-831.

Mondal, M., Trivedy, K., and Kumar, S. N. 2007. The silk proteins, sericin and fibroin in silkworm, Bombyx mori Linn., A review. Caspian J. Environ. Sci. 5:63-76.

Mossadegh, M. S. 1978. Mechanism of secretion of the contents of the mandibular glands of Plodia interpunctella larvae. Physiol. Entomol. 3:335-340.

Musser, R. O., Hum-Musser, S. M., Eichenseer, H., Peiffer, M., Ervin, G., Murphy, J. B., and Felton, G. W. 2002. Herbivory: Caterpillar saliva beats plant defences. Nature 416:599-600.

Musser, R. O., Farmer, E., Peiffer, M., Williams, S. A., and Felton, G. W. 2006. Ablation of caterpillar labial salivary glands: Technique for determining the role of saliva in insect-plant interactions. J. Chem. Ecol. 32:981-992.

Parthasarathy, R., and Gopinathan, K. P. 2005. Comparative analysis of the development of the mandibular salivary glands and the labial silk glands in the mulberry silkworm, Bombyx mori. Gene Expr. Patterns 5:323-339.

Peiffer, M., and Felton, G. W. 2005. The host plant as a factor in the synthesis and secretion of salivary glucose oxidase in larval Helicoverpa zea. Arch. Insect Biochem. Physiol. 58:106-113.

Peiffer, M., and Felton, G. 2009. Do caterpillars secrete "oral secretions"? J. Chem. Ecol. 35:326-335.

Reymond, P., Weber, H., Damond, M., and Farmer, E. E. 2000. Differential gene expression in response to mechanical wounding and insect feeding in Arabidopsis. Plant Cell 12:707-720.

Riberiro, J. M. C. 1995. Insect saliva: Function, biochemistry, and physiology. Pages 74-98 in: Regulatory Mechanisms in Insect Feeding. R. F. Chapman and G. D. Boer, eds. Chapman \& Hall, New York.

Ritchie, S. W., Hanway J. J., and Benson, G. O. 1986. How a corn plant develops. Iowa State Univ. Sci. Technol. Coop. Ext. Serv. Rep. 48, Ames.

Rohrmeier, T., and Lehle, L. 1993. WIP1, a wound-inducible gene from maize with homology to Bowman-Birk proteinase inhibitors. Plant Mol. Biol. 22:783-792

Schäfer, M., Fischer, C., Meldau, S., Seebald, E., Oelmüller, R., and Baldwin, I. T. 2011. Lipase activity in insect oral secretions mediates defense responses in Arabidopsis. Plant Physiol. 156:1520-1534.

Schaller, A., and Stintzi, A. 2009. Enzymes in jasmonate biosynthesisstructure, function, regulation. Phytochemistry 70:1532-1538.

Schmelz, E. A., Alborn, H. T., and Tumlinson, J. H. 2001. The influence of intact-plant and excised-leaf bioassay designs on volicitin- and jasmonic acid-induced sesquiterpene volatile release in Zea mays. Planta 214:171-179.

Schmelz, E. A., Carroll, M. J., LeClere, S., Phipps, S. M., Meredith, J., Chourey, P. S., Alborn, H. T., and Teal, P. E. A. 2006. Fragments of ATP synthase mediate plant perception of insect attack. Proc. Natl. Acad. Sci. U.S.A. 103:8894-8899.

Schmelz, E. A., Engelberth, J., Alborn, H. T., Tumlinson, J. H., and Teal, P. E. A. 2009. Phytohormone-based activity mapping of insect herbivoreproduced elicitors. Proc. Natl. Acad. Sci. U.S.A. 106:653-657.

Shivaji, R., Camas, A., Ankala, A., Engelberth, J., Tumlinson, J. H., Williams, W. P., Wilkinson, J. R., and Luthe, D. S. 2010. Plants on constant aert: Elevated levels of jasmonic acid and jasmonate-induced transcripts in caterpillar-resistant maize. J. Chem. Ecol. 36:179-191.

Sorensen, G. S., Cribb, B. W., Merritt, D., Johnson, M.-L., and Zalucki, M. P. 2006. Structure and ultrastructure of the silk glands and spinneret of Helicoverpa armigera (Hübner) (Lepidoptera: Noctuidae). Arthropod Struct. Dev. 35:3-13. 
Spiteller, D., Pohnert, G., and Boland, W. 2001. Absolute configuration of volicitin, an elicitor of plant volatile biosynthesis from lepidopteran larvae. Tetrahedron Lett. 42:1483-1485.

Tamayo, M. C., Rufat, M., Bravo, J. M., and San Segundo, B. 2000. Accumulation of a maize proteinase inhibitor in response to wounding and insect feeding, and characterization of its activity toward digestive proteinases of Spodoptera littoralis larvae. Planta 211:62-71.

Thivierge, K., Prado, A., Driscoll, B. T., Bonneil, E. R., Thibault, P., and Bede, J. C. 2010. Caterpillar- and salivary-specific modification of plant proteins. J. Proteome Res. 9:5887-5895.

Tian, D., Peiffer, M., Shoemaker, E., Tooker, J., Haubruge, E., Francis, F., Luthe, D. S., and Felton, G. 2012. Salivary glucose oxidase from caterpillars mediates the induction of rapid and delayed-induced defenses in the tomato plant. PLoS One 7:e36168. Published online.

Ton, J., D’Alessandro, M., Jourdie, V., Jakab, G., Karlen, D., Held, M., Mauch-Mani, B., and Turlings, T. C. J. 2007. Priming by airborne signals boosts direct and indirect resistance in maize. Plant J. 49:1626.

Wang, C., Zien, C. A., Afitlhile, M., Welti, R., Hildebrand, D. F., and Wang,
X. 2000. Involvement of phospholipase D in wound-induced accumulation of jasmonic acid in Arabidopsis. Plant Cell 12:2237-2246.

Williams, W., Buckley, P., and Davis, F. 1989. Combining ability for resistance in corn to fall armyworm and southwestern corn borer. Crop Sci. 29:913-915.

Williams, W. P., Davis, F. M., and Windham, G. L. 1990. Registration of Mp708 germplasm line of maize. Crop Sci. 30:757-.

Wroniszewska, A. 1966. Mandibular glands of the wax moth larva, Galleria mellonella (L.). J. Insect Physiol. 12:509-514.

Wu, J., and Baldwin, I. T. 2010. New insights into plant responses to the attack from insect herbivores. Annu. Rev. Genet. 44:1-24.

Wu, S., Peiffer, M., Luthe, D. S., and Felton, G. W. 2012. ATP hydrolyzing salivary enzymes of caterpillars suppress plant defenses. PLoS One 7:e41947. Published online.

Yoshinaga, N., Aboshi, T., Ishikawa, C., Fukui, M., Shimoda, M., Nishida, R., Lait, C., Tumlinson, J., and Mori, N. 2007. Fatty acid amides, previously identified in caterpillars, found in the cricket Teleogryllus taiwanemma and fruit fly Drosophila melanogaster larvae. J. Chem. Ecol. 33:1376-1381. 